\title{
FUNCTION THEORETIC METHODS IN THE THEORY OF BOUNDARY VALUE PROBLEMS FOR GENERALIZED METAHARMONIC FUNCTIONS ${ }^{1}$
}

\author{
BY DAVID COLTON AND ROBERT P. GILBERT
}

Communicated by Wolfgang Wasow, March 17, 1969

1. Introduction. Although existence and uniqueness theorems for linear elliptic partial differential equations in a domain $D$ with coefficients continuous in $D$ have been known for some time, similar results for equations whose coefficients have singularities in the domain under consideration are practically unknown. Recently attention has been given to a class of singular equations which appear frequently in both pure and applied mathematics and are known as generalized axially symmetric partial differential equations [3], [5], [8]. Just as a thorough knowledge of the Laplace and Helmholtz equation guided the attack on linear elliptic equations with continuous coefficients, it is hoped that a better understanding of generalized axially symmetric equations will give insight towards developing a theory of elliptic equations with singular coefficients.

We wish to announce in this note a uniqueness theorem for the exterior Dirichlet problem for the generalized axially symmetric metaharmonic equation

$$
L_{\lambda_{\varepsilon}}[u] \equiv \frac{\partial^{2} u}{\partial x_{1}^{2}}+\cdots+\frac{\partial^{2} u}{\partial x_{n}^{2}}+\frac{\partial^{2} u}{\partial \rho^{2}}+\frac{s}{\rho} \frac{\partial u}{\partial \rho}+\lambda^{2} u=0
$$

where $s>-1, s \neq 0$, and $\lambda>0$ [5]. This is the first time a uniqueness theorem has been obtained for a singular elliptic partial differential equation in more than two variables whose coefficients are singular in its domain of definition, such work in the present case having been delayed due to an insufficient knowledge of certain areas of the theory of several complex variables. Our result depends on first using the Hadamard-Gilbert Theorem [5] to solve the classical expansion problem for Appell series and to then apply this along with Vitali's theorem for several complex variables [6] to obtain the desired uniqueness theorem.

${ }^{1}$ This research was supported in part by the Air Force Office of Scientific Research through grant AFOSR-1206-67. 
2. The expansion problem for Appell series. An Appell series is a series of the form

$$
\sum_{M} a_{M} V_{M}^{(s)}(\xi) \equiv \sum_{\mu=0}^{\infty} \sum_{m=\mu} a_{M} V_{M}^{(s)}(\xi) ; \quad s>-1, s \neq 0
$$

where the polynomials

$$
V_{\boldsymbol{M}}^{(\boldsymbol{s})}(\xi) \equiv V_{\boldsymbol{M}}^{(s)}\left(\xi_{1}, \cdots, \xi_{n}\right) ; \quad M=\left(m_{1}, m_{2}, \cdots, m_{n}\right)
$$

are uniquely defined by the generating function

$$
\left(1-2(\alpha, \xi)+\|\alpha\|^{2}\right)^{-(n+s-1) / 2}=\sum \alpha^{M} V_{M}^{(s)}(\xi) .
$$

Here $(\alpha, \xi) \equiv \sum_{i=1}^{n} a_{i} \xi_{i},\|\alpha\|^{2}=(\alpha, \alpha), \alpha^{M} \equiv \alpha_{1}^{m_{1}} \cdots \alpha_{n}^{m_{n}}, m=|M|$ $=m_{1}+m_{2}+\cdots+m_{n}$ and the summation in equation (2), (4), and in what follows, is meant to be an $n$-fold sum over all indices from zero to infinity. We also need the related polynomials

$$
U_{M}^{(s)}(\xi) \equiv U_{M}^{(s)}\left(\xi_{1}, \xi_{2}, \cdots, \xi_{n}\right)
$$

which are defined by the generating function

$$
\left\{[(\alpha, \xi)-1]^{2}+\|\alpha\|^{2}\left(1-\|\xi\|^{2}\right)\right\}^{-8 / 2}=\sum \alpha^{M} U_{M}^{(s)}(\xi) .
$$

These polynomials satisfy the biorthogonality relation

$$
\begin{aligned}
\int_{S(0,1)}\left(1-\|\xi\|^{2}\right)^{(s-1) / 2} V_{M}^{(s)}(\xi) U_{L}^{(s)}(\xi) d^{n} \xi \\
\quad=\delta_{L M} \frac{2 \pi^{n / 2} \Gamma(s / 2+1)(s) m}{(2 m+n+s-1) \Gamma(n / 2+s / 2-1 / 2) M !},
\end{aligned}
$$

$\delta_{L M} \equiv \delta_{l_{1} m_{1}} \cdots \delta_{l_{n} m_{n}}, M !=m_{1} ! \cdots m_{n}$ !. Here $S(0,1)$ is the real solid $n$ dimensional ball $\{\xi \mid\|\xi\| \leqq 1\}$. For more information concerning the polynomials $V_{M}^{(s)}(\xi)$ and $U_{M}^{(s)}(\xi)$ see [1]. We are interested in the classical expansion problem for analytic functions of $n$ complex variables in terms of the polynomials $V_{M}^{(s)}(\xi)$ viz. if $f(\xi)$ is analytic on $S(0,1)$ can it be expanded in a series of the form (2) and if so what can be said about the region of convergence?

THEOREM 1. Let $f(\xi)$ be an analytic function of $n$ complex variables in some neighborhood $\tilde{\eta}$ of the unit ball $S(0,1)$. Then $f(\xi)$ can be expanded in an Appell series,

$$
f(\xi)=\sum a_{M} V_{M}^{(s)}(\xi) ; \quad s>-1, s \neq 0
$$


which converges uniformly for $\xi \in \Im \equiv \eta\{S(0,1)\} \cap \eta^{*}\{S(0,1)\}$ with $\eta\{S(0,1)\} \subset \tilde{\eta}, \eta^{*} \equiv\left\{\xi \mid \xi^{*} \in \eta\right\}$ ( ${ }^{*}$ denotes complex conjugation), and where the coefficients are given by the formula

$$
a_{M}=h_{M}^{*} \int_{S(0,1)}(1-\|\xi\|)^{(s-1) / 2} f(\xi) U_{M}^{(s)}(\xi) d^{n} \xi
$$

with

$$
\dot{h}_{M}^{:}=\frac{(2 m+n+s-1) \Gamma\left(\frac{1}{2}[n+s-1]\right) M !}{2 \pi^{n / 2} \Gamma(s / 2+1)(s)_{m}} .
$$

OUTLINE OF PROOF. The technique used is to develop an integral operator approach to the method of generating kernels [2]. We first define a new function $F(\zeta)$ defined by

(8) $\quad F(\zeta)=O f(\xi)=\left(\frac{1}{2 \pi i}\right)^{n} \int_{S(0,1)} K_{1}(\zeta, \xi) f(\xi) d^{n} \xi=\sum a_{M} \zeta^{M}$

where

$$
\begin{aligned}
K_{1}(\zeta, \xi) \equiv & \frac{\Gamma\left(\frac{1}{2}[n+s-1]\right)\left(1-\|\xi\|^{2}\right)^{(s-1) / 2}}{2 \pi^{n / 2} \Gamma(s / 2+1)} \\
& \cdot \sum \frac{2 m+n+s-1}{(s)_{m}} M ! U_{M}^{(s)}(\xi) \zeta^{M} .
\end{aligned}
$$

From the Weierstrass comparison theorem [5] and the generating function expansion (6) it can be shown that equation (8) defines an analytic function of $\zeta$ for $\zeta \in \Delta(0,1)$. Here $\Delta(0,1)$ denotes the open unit ball in the complex $\zeta$ space. We next define the inverse operator $O^{-1}$ by

$$
\begin{aligned}
f(\xi) & =O^{-1} F(\zeta)=\left(\frac{1}{2 \pi i}\right)^{n} \int_{\Gamma} K_{2}(\xi, \zeta) F(\zeta) \frac{d^{n} \zeta}{\zeta} \\
& =\sum a_{M} V_{M}^{(s)}(\xi)
\end{aligned}
$$

where

$$
K_{2}(\xi, \zeta) \equiv \sum \zeta^{-M} V_{M}^{(s)}(\xi)=\left(1-2\left(\frac{1}{\zeta}, \xi\right)+\left\|\frac{1}{\zeta}\right\|^{2}\right)^{-(n+s-1) / 2}
$$

and $\Gamma=\prod_{i=1}^{n} \Gamma_{i}$ where the $\Gamma_{i}$ are chosen such that $\Gamma$ lies outside a sufficiently large hypersphere $\Delta(0, R)$. By using the HadamardGilbert theorem [5] and the fact that $f(\xi)$ is analytic in $\tilde{\eta}$, one can 
conclude from (8) that $F(\zeta)$ must in fact be an analytic function in some complex neighborhood of $\overline{\Delta(0,1)}$ on its principal sheet of definition. Using, this fact in conjunction with the series representation (8) for $F(\zeta)$ shows that the series (10) converges uniformly in some complex neighborhood of $S(0,1)$ and agrees with $f(\xi)$ there. The formula for the coefficients is arrived at through use of the biorthogonality relation (7).

3. The uniqueness theorem. Since the plane $\rho=0$ is a singular curve of the regular type with indices 0 and $1-s$ [4] there always exist solutions of equation (1) which are real analytic on some portion of the axis $\rho=0$, and if $s>-1, s \neq 0$, such solutions can be continued across the axis as an even function of $\rho$. Hence each such analytic solution is analytic in a domain $D$ that is symmetric with respect to the axis $\rho=0$ and can therefore be expressed as $u(x, \rho)=\tilde{u}(r, \xi)$ where $r, \xi=\left(\xi_{1}, \cdots, \xi_{n}\right)$ are hyper-zonal coordinates.

Theorem 2. Assume $s>-1, s \neq 0$, and let $u(x, \rho)=\tilde{u}(r, \xi)$ be a real analytic solution of $L_{\lambda_{s}}[u]=0$ in $\mathfrak{F}$ where $\mathfrak{F}$ is the exterior of a normal domain $D$ which is symmetric with respect to the axis $\rho=0$. Let $\tilde{u}(r, \xi)$ $\in C^{2}(\mathfrak{F})$ and for each fixed $r, a \leqq r<\infty$ (where $a$ is such that $r=a$ contains $D)$ assume that $\tilde{u}(r, \xi)$ is an analytic function of $\xi$ in a domain $\Im$ in the complex $\xi$ space containing the (real) closed unit ball $S(0,1)$, where $\Im$ is symmetric with respect to conjugation and independent of $r$. If

(i) there exists a positive constant $M$ such that

$$
r^{(n+s) / 2}|\tilde{u}(r, \xi)| \leqq M \quad \text { for } a \leqq r<\infty, \quad \xi \in \Im,
$$

(ii) $\lim _{r \rightarrow \infty} \int_{S(0,1)} r^{n+*}\left(1-\|\xi\|^{2}\right)^{(8-1) / 2}|\partial \tilde{u} / \partial r-i \lambda \tilde{u}|^{2} d S=0$,

(iii) $\tilde{u}(r, \xi)=0$ on the boundary of $D$

then $\tilde{u}(r, \xi) \equiv 0$.

REMARK. If conditions (i) and (ii) of the theorem are not imposed there will exist eigenfunctions of equation (1) and we cannot expect a unique solution to exist.

OUtLINE OF PROOF. Using Theorem 1 we expand $\tilde{u}(r, \xi)$ for each fixed $r \geqq a$ in an Appell series

$$
\tilde{u}(r, \xi)=\sum a_{M}(r) V_{M}^{(s)}(\xi) ; \quad \xi \in \Im .
$$

From the biorthogonality property (7) it can be shown that

$$
a_{M}(r)=r^{-1 / 2(n+\sigma-1)} Z_{\mu+1 / 2(n+s-1)}(\lambda r)
$$

where $Z_{\nu}(\lambda r)$ denotes a cyclinder function of order $\nu$. Applying Green's formula and condition (ii) gives 


$$
\lim _{r \rightarrow \infty} \int_{S(0,1)} r^{n+8}\left(1-\|\xi\|^{2}\right)^{(8-1) / 2}|u|^{2} d^{n} \xi=0 .
$$

If the polynomials $V_{M}^{(s)}(\xi)$ satisfied Parseval's relation we could now proceed with the proof by following the approach used by Vekua in [7]. Since this is not the case we resort to techniques first used by Colton in [3]. Define

$$
g(r, \xi) \equiv \tilde{u}(r, \xi) \tilde{u}^{*}\left(r, \xi^{*}\right),
$$

and observe that $g(r, \xi)$ is an analytic function of $\xi$ which agrees with $|u(r, \xi)|^{2}$ for $\xi$ real valued. From condition (i) of the theorem it can be shown that $r^{n+s}|g(r, \xi)|$ is uniformly bounded for $a \leqq r<\infty, \xi \in \Im$. Now define for fixed $r$ and variable $\xi$

$$
F_{r}(\xi) \equiv \int_{0}^{\xi} r^{n+s}\left(1-\|\xi\|^{2}\right)^{((s-1) / 2)} g(r, \xi) d^{n} \xi
$$

and note that $F_{r}(\xi)$ is analytic in $\Delta(0, \epsilon)$ (if $\epsilon$ is chosen sufficiently small) and for $r \geqq a, \xi \in \Delta(0, \epsilon),\left|F_{r}(\xi)\right|$ is uniformly bounded. By applying a version of Vitali's theorem for several complex variables [6], it can be shown using equations (13) and (15) that

$$
\lim _{r \rightarrow \infty} r^{(n+s) / 2} \tilde{u}(r, \xi)=0 ; \quad \xi \in S(0,1) .
$$

Theorem 1, equation (12), and the asymptotic behavior of cylinder functions (c.f. [7]) now shows that $a_{M}(r)=0$ for every $M$ and hence $\tilde{u}(r, \xi) \equiv 0$.

\section{REFERENCES}

1. P. Appell and J. DeFeret, Fonctions hypergéometrigues et hypersphériques, Polynomes d'Hermite, Gauthier-Villars, Paris, 1926.

2. R. Boas and R. Buck, Polynomial expansions of analytic functions, SpringerVerlag, Berlin, 1964.

3. D. Colton, Analytic theory and uniqueness theorems for the generalized axially symmetric Schrödinger equation, J. Differential Equations (to appear).

4. A. Erdélyi, The analytic theory of systems of partial differential equations, Bull. Amer. Math. Soc. 57 (1951), 339-353.

5. R. Gilbert, Function theoretic methods in partial differential equations, Academic Press, New York, 1969.

6. R. Gunning and H. Rossi, Analytic functions of several complex variables, Prentice-Hall, Englewood Cliffs, N.J., 1965.

7. I. Vekua, New methods for solving elliptic equations, Wiley, New York, 1968.

8. A. Weinstein, Singular partial differential equations and their applications, Proc. Sympos. in Fluid Dynamic and Appl. Math., Gordon and Breach, New York, 1961.

INDIANA UNIVERSITY, BLOOMINGTON, INDIANA 47405 